\title{
Anticipatory contrast as a measure of time horizons in the rat: Some methodological determinants
}

\author{
GARY A. LUCAS, DONALD J. GAWLEY, and WILLIAM TIMBERLAKE \\ Indiana University, Bloomington, Indiana
}

\begin{abstract}
In three experiments, the time horizon over which the rat evaluates alternative feeding sources was investigated. The time horizon was measured by the suppression of intake of one incentive (a $0.15 \%$ saccharin solution) when a preferred alternative incentive (a $32 \%$ sucrose solution) was available but delayed. In Experiment 1, we found a direct function between the amount of saccharin intake and the delay time before access to $32 \%$ sucrose. Compared with intake for a saccharin-only control, saccharin intake was suppressed before 4-min and 16-min sucrose delays, but not before a 32-min delay. Because previous work (Flaherty \& Checke, 1982) had reported suppression before a delay of nearly $32 \mathrm{~min}$, in the subsequent experiments we examined factors that might account for this difference. In Experiment 2, we found that saccharin intake was suppressed before a 32-min delay interval when saccharin and sucrose solutions were presented in a bright-novel test environment but not when the same solutions were presented in the home cage. In Experiment 3, we found that the time between testing and subsequent postsession feeding could also affect the suppression of saccharin intake. Saccharin intake was suppressed when access to $32 \%$ sucrose was delayed by $32 \mathrm{~min}$ and the test situation was followed by immediate postsession feeding, but not when postsession feeding was delayed by $90 \mathrm{~min}$. These results thus extend estimates of the rat's time horizon to at least $32 \mathrm{~min}$, but indicate that the effective time horizon can vary, depending on the test situation.
\end{abstract}

A critical question for understanding an animal's foraging behavior involves the period of time over which resources are evaluated as alternatives. This time period has been termed the time horizon of the animal (Kamil \& Roitblat, 1985; Lea, 1981). The time horizon question is particularly important for theories of optimal foraging (see Pyke, Pulliam, \& Charnov, 1977), because it represents the temporal limits within which an animal can be expected to optimize behavior. Recently, we reported data that placed the rat's time horizon around $16 \mathrm{~min}$ (Timberlake, Gawley, \& Lucas, 1987). This estimate was obtained by providing rats with access to an increasingly costly (i.e., a depleting) patch, which was followed, after a delay, by access to an abundant patch with minimal cost. The rats suppressed their food intake in the depleting patch only when the less costly patch was delayed by $16 \mathrm{~min}$ or less.

The aim of the present study was to determine the generality of our previous results by using a different procedure to estimate the rat's time horizon. It may be that the response costs related to a food are evaluated across a time frame different from that for the evaluation of food quality (e.g., its hedonic or caloric value). Some evidence for this possibility has been provided by Flaherty and Checke

This research was supported by National Institute of Health Grant MH37892. We wish to thank Carey Jones and Dean Wallace for help in collecting data. The authors' mailing address is: Psychology Department, Indiana University, Bloomington, IN 47401.
(1982), who reported that the intake of a $0.15 \%$ saccharin solution was suppressed when it was followed by a $32 \%$ sucrose solution after delays of up to $30 \mathrm{~min}$. In addition, other factors that suppress or arouse feeding motivation might also be expected to alter the time period over which future food can affect current performance.

The first study presented here was an attempt to replicate Flaherty and Checke's (1982) finding that rats would suppress their intake of a saccharin solution when it was followed by access to a $32 \%$ sucrose solution at delays of up to $32 \mathrm{~min}$. Upon failure to find suppression with the 32-min delay, in the subsequent studies we examined possible methodological factors that might have accounted for the failure to replicate here, as well as for the failure of Flaherty and Checke (1982) to replicate this effect on one occasion. In Experiment 2, we manipulated the novelty of the test environment. In Experiment 3, we examined the temporal relationship between the time of testing and the subsequent daily feeding period.

\section{EXPERIMENT 1}

When animals are repeatedly exposed to two solutions, one of which regularly precedes a more preferred solution, a suppression of intake of the first solution may develop. This effect has been called anticipatory contrast. The contrast obtained in this procedure appears to depend on learning, in that it cannot be accounted for by immediate sensory mechanisms, it develops gradually along with 
experience, and it is sensitive to the delay and hedonic value of the second incentive (Flaherty \& Checke, 1982; Flaherty \& Rowan, 1985, 1986). In effect, the mechanisms involved here appear to decrease the value of the first food source when anticipation of a more preferred future food occurs.

The anticipatory contrast paradigm differs in several important ways from the paradigm used by Timberlake et al. (1987) to assess the effects of future food. In that study, the rats were faced with the problem of expending a high work cost to obtain food in one patch, as opposed to waiting for the same food available at a lower cost in a second patch. That is, the two patches differed in cost and density (in time), but the same food stuff was available in both patches. In the anticipatory contrast paradigm, the cost of obtaining food in each access period is the same and minimal, but the access periods vary in terms of the quality of food available. This paradigm also appears to rule out the possibility, raised by Timberlake et al. (1987), that the suppression of intake in the first patch results from a competition between eating in the first patch and inspecting the second patch for the availability of food. In the present procedure, the alternative incentive items are presented in the same location but at different times.

In two of three studies using 30-min delays, Flaherty and Checke (1982) found that rats would suppress intake of $0.15 \%$ saccharin solution when a more preferred $32 \%$ sucrose solution was available after a 30-min delay. This interval is greater than the 16-min interval for suppression that we reported when the density of the food was varied across conditions (Timberlake et al., 1987). In the present study, we therefore attempted to replicate the results of Flaherty and Checke, with particular attention to the degree of suppression obtained around the 30-mindelay interval. As in the Flaherty and Checke procedure, we used $0.15 \%$ saccharin for the first solution and $32 \%$ sucrose for the second solution. The delays between solutions were 4,16 , and $32 \mathrm{~min}$. The control group received one access period of $0.15 \%$ saccharin solution and no second solution.

\section{Method}

Subjects. Thirty-six 120-150-day-old experimentally naive female rats served. The animals were bred locally from SpragueDawley stock. Their individual weights ranged from about 240-300 g. The animal colony was maintained on a 12:12 h light:dark cycle. Water was available continuously in the home cages.

Approximately 8 days before the beginning of testing, the subjects were isolated in individual cages and their daily food was restricted ( $7 \mathrm{~g}$ per day) until each subject was reduced to $85 \%$ of its ad-lib body weight. During this period all subjects were weighed and fed daily at the time of subsequent testing. The subjects were then divided into four groups of 9 animals each, equated for body weight.

Apparatus. A standard cage rack was modified so that the animals' home cages could be placed in the rack and the animals could be transported in groups to the testing room. Each cage mea- sured $24 \mathrm{~cm}$ long $\times 19 \mathrm{~cm}$ wide $\times 18 \mathrm{~cm}$ high. The back wall and both side walls of the cage were made from stainless steel sheeting. The front and bottom of the cage was made of stainless steel wire mesh. The solutions were placed in individual drinking wells made from 50-ml beakers mounted in metal holders. The holders attached to the rack and held the beakers tilted at a $40^{\circ}$ angle, to make them easily accessible to the rats. The holders were lowered into the cages by the experimenter and fastened in place to prevent movement and spillage. The testing room was directly vented to the outside by an overhead exhaust fan. During testing the room was dimly lit by a single $25-\mathrm{W}$ red bulb mounted about $2 \mathrm{~m}$ from the test rack.

Procedure. The four groups of rats were assigned to one of three delay conditions (4 $\mathrm{min}, 16 \mathrm{~min}$, or $32 \mathrm{~min}$ ), or to one control condition. Testing consisted of one trial per day for 12 consecutive days. On each day, the rats were removed from their cages for weighing and then returned to the cages, which were placed in the modified rack and transported to the test room. Upon arrival in the room, the rats were left for a 10 -min accommodation period.

During the accommodation period, the test solutions were measured out and placed in individual drinking wells. Additionally, across the first 4 days of testing, each animal that had not consumed at least $0.4 \mathrm{ml}$ of saccharin on the previous day received a $0.1-\mathrm{ml}$ drop of saccharin solution. The saccharin solution was applied to the mouth with a plastic 1-cc syringe. Thus, all animals received a drop of saccharin on the first testing day, and all continuing " nondrinkers" received similar treatment as needed over the next 3 days. This forced exposure to saccharin occurred about $5 \mathrm{~min}$ before the beginning of the test trial, and ensured that all animals came in contact with the saccharin. Pilot data indicated that without forced exposure, about $15 \%$ of the animals would take a week or longer to sample a solution when given only $5 \mathrm{~min}$ of access to the solution per day. In this and the following studies, any subject that failed to consume at least $0.4 \mathrm{ml}$ of saccharin after the first 4 days was removed from the study. This precaution was used to ensure that the acquisition curves remained roughly parallel. One animal was excluded from the 4-min group in the present experiment, for failure to drink.

At the end of the $10-\mathrm{min}$ accommodation period, a $10-\mathrm{ml}$ volume of $0.15 \%$ saccharin was placed into the rat's cage and left there for $5 \mathrm{~min}$. Following the 5 -min access period, the saccharin solution was removed, and the delay period $(4,16$, or $32 \mathrm{~min})$ began. At the end of the delay period, the $32 \%$ sucrose solution was placed in the rat's cage for $5 \mathrm{~min}$. Following removal of the sucrose solutions, the rats were returned to the colony. The subjects in the control group received $10 \mathrm{ml}$ of saccharin access for $5 \mathrm{~min}$, remained in the test room for a 32-min delay period, and were then returned to the colony without receiving a second access period. A daily feeding of Purina lab chow sufficient to maintain each subject at $85 \%$ body weight was provided $90 \mathrm{~min}$ after the end of each test session.

Saccharin and sucrose solutions were mixed at 3-day intervals and were kept refrigerated at $4^{\circ} \mathrm{C}$ until they were measured out each day. The solutions were therefore cool when they were presented at the time of testing. The saccharin solution was mixed from a commercially available $2.33 \%$ stock solution (Pillsbury Sweet-10), by diluting it with tap water. The sucrose solution was mixed from commercially available cane sugar and tap water to form a $32 \%$ solution by weight (e.g., $47.1 \mathrm{~g}$ sucrose $/ 100 \mathrm{~g}$ water).

The test solutions were provisioned so that there was more solution available than each rat drank in the 5-min test period. For the saccharin solutions this was always $10 \mathrm{ml}$. For the sucrose solution, we began with $10 \mathrm{ml}$ but increased the amount to 12 or $15 \mathrm{ml}$ as necessary. Consumption was measured by using $10-\mathrm{ml}$ graduated cylinders to provision the drinking wells and to measure the amount remaining after testing. The wells were always washed and 
dried between groups. Loading of the wells and measurement of solutions was carried out in another room, which was on a separate ventilation system. Each solution remained outside the test room until it was needed. These precautions ensured that olfactory cues from the second solution were not present when the first solution was tested. Waxed paper was placed under each rat's cage at the time of testing to collect any spillage. Spillage from the wells was rare, but when it did occur, droplets could be easily recovered from the waxed paper, by using a small syringe.

\section{Results and Discussion}

The amount of $0.15 \%$ saccharin intake in the first access period was directly related to the delay time before access to the $32 \%$ sucrose solution. Figure 1 shows the mean saccharin intake for each group, averaged across the last 2 days of testing. A one-way ANOVA on these measures indicated significant differences in saccharin intake among the four groups $[F(3,31)=10.75, p<.01]$. Post hoc comparisons (Newman-Keuls) showed no significant difference in saccharin intake between the 32-min and control groups. However, saccharin intake in the 4-min and 16-min groups was significantly less than in the control group ( $p<.01$ and .05 , respectively).

The amount of sucrose intake in the second access period did not vary significantly as a function of the delay $[M=9.8 \mathrm{ml}, S E=0.68 ; M=8.9 \mathrm{ml}, S E=0.66$; and $M=9.9 \mathrm{ml}, S E=0.71$, respectively, for the $4-, 16-$, and $32-\mathrm{min}$ delay conditions; $F(2,23)=1.88$, $p>.17]$.

These findings suggest that the rats learned the relationship between the two access periods, and that they reduced their saccharin intake in the first period in anticipation of the availability of sucrose in the second. The results thus replicate those of Flaherty and Checke (1982) for the 4-min and 16-min delay groups, but fail to demonstrate suppression of saccharin intake with a 32-min delay. Instead, the time period over which suppression of intake was observed as the rats anticipated future food was similar to that reported by Timberlake et al. (1987) when the density of a food was varied across access

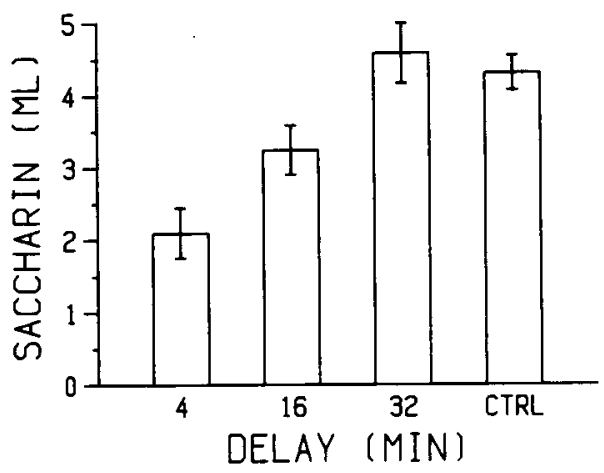

Figure 1. The mean intake of $0.15 \%$ saccharin solution for the last 2 days of testing across groups in Experiment 1. Delay times indicate the time from the end of the saccharin access period to the presentation of $32 \%$ sucrose solution in the second access period. The control group did not receive a second access period. Bars indicate the standard error of the mean for each group. periods. This observation suggests that variations in the type of food available from patch to patch may be evaluated in a similar manner as variations in the density of food.

\section{EXPERIMENT 2}

In Experiment 1, we found suppression of saccharin intake with shorter intervals but failed to find evidence of suppression when the delay to the second access period was $32 \mathrm{~min}$. However, as we noted above, Flaherty and Checke (1982) only found suppression of intake with the 30-min interval in two of three studies. One possibly important difference between the procedures of Flaherty and Checke (1982) and those in the present Experiment 1 was the amount of novelty in the test situation. Flaherty and Checke tested their animals in chambers that differed from their home cages, while we tested our animals in a more familiar home cage environment. Because novelty may increase fearfulness, perhaps the rat is more cautious about feeding in a novel environment, and thus more likely to suppress intake in a novel testing situation, than in a more familiar environment like that in Experiment 1. The possibility that the animals are more likely to suppress intake under fear-provoking circumstances might also explain why animals with previous experimental history might show less suppression (see Flaherty \& Checke, 1982, Experiment 3), because experienced animals might be less fearful than naive animals when placed in a novel test environment.

Experiment 2 was therefore conducted to test the hypothesis that novel characteristics of the test environment can influence the time horizon over which rats show suppression of intake in the anticipatory contrast paradigm. We compared saccharin intake in two test situations, one novel and presumably more fear-provoking, and one very familiar. For the novel situation we removed the animals from their home cages, transported them to a brightly illuminated room, and placed them in individual plastic tubs where we measured their consumption of saccharin and sucrose solutions. For the familiar situation, we left the animals in the rat colony in their home cages and measured the saccharin and sucrose consumption there. In addition, in order to control for the effects of presenting two access periods for the experimental group, as opposed to only one for the control group in Experiment 1 , we provided the control groups in the present procedure with two access periods. In the first period a $0.15 \%$ saccharin solution was presented; in the second, water alone was presented.

\section{Method}

Subjects. Forty-eight 120-150-day-old experimentally naive female rats were maintained and treated as in Experiment 1.

Apparatus. For the familiar (home) test environment, the rats were tested in the animal colony in their home cages. The home cages measured $24 \mathrm{~cm}$ long $\times 19 \mathrm{~cm}$ wide $\times 18 \mathrm{~cm}$ high and were identical to the cages used in Experiment 1. The same drinking wells as were described under Experiment 1 were used. 
For the unfamiliar (novel) test environment, the rats were tested in $25 \mathrm{~cm}$ long $\times 22 \mathrm{~cm}$ wide $\times 20 \mathrm{~cm}$ high polypropylene plastic tubs-hamster cages with stainless steel wire tops. A metal ridge was attached near the top of one side wall of the plastic tubs to allow the drinking wells to be attached to the plastic tubs.

Procedure. The animals were divided into four groups-two control groups and two experimental groups-equated for body weight. One pair of control and experimental groups was tested in the novel environment; the other pair was tested in the home cages.

After weighing and a 10-min accommodation period, the subjects in each group were given a 5-min period of access to $10 \mathrm{ml}$ of a $0.15 \%$ saccharin solution. A 32-min delay was timed from the end of the saccharin period to the beginning of the second access period. The subjects in the experimental groups then received 5 min of access to a $32 \%$ sucrose solution. The subjects in the control groups received only water during the second access period. All other procedures were identical to those in Experiment 1. Daily feedings were provided $90 \mathrm{~min}$ after the end of each test session, to maintain $85 \%$ body weight.

\section{Results and Discussion}

The amount of $0.15 \%$ saccharin intake for each group is summarized in Figure 2, which shows the means averaged across the last 2 days of testing. An ANOVA for a $2 \times 2$ factorial design was used to compare saccharin intake across the two test locations (home and novel) and the two solutions (water and $32 \%$ sucrose) available in the second period. The analysis indicated a significant difference in saccharin intake for test location $[F(1,34)$ $=10.37, p<.01]$, a nonsignificant difference for test solution $[F(1,34)=2.40, p=.13]$, and a significant location $\times$ solution interaction $[F(1,34)=7.83, p<.01]$. A simple main effects comparison of locations with respect to solution indicated no significant difference in saccharin intake between the home and novel locations for water, but a significant difference between home and novel locations for $32 \%$ sucrose.

The difference in sucrose intake between the home $(M$ $=9.1 \mathrm{ml}, S E=.29)$ and novel $(M=8.0 \mathrm{ml}, S E=0.43)$ conditions approached significance $[F(1,20)=4.31, p=$ $.051]$. Although there was occasional sampling of the water early in training, water intake in both the home ( $M$

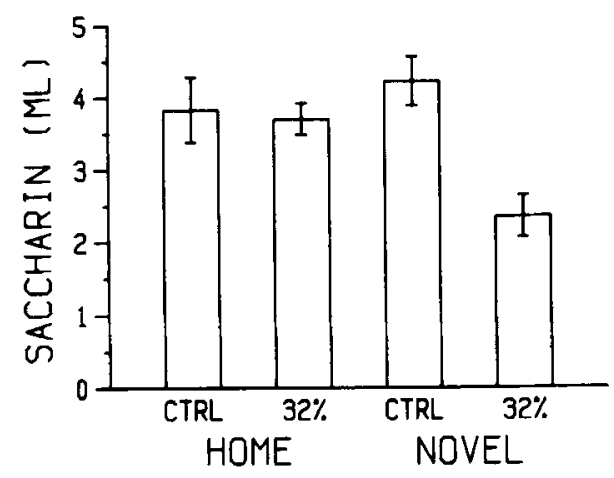

Figure 2. The mean intake of $0.15 \%$ saccharin solution for the last two days of testing across groups in Experiment 2. One pair of groups (home) was tested in the animal colony in their home cages. The second pair (novel) was tested in a brightly illuminated novel environment. Bars indicate the standard error of the mean for each group.
$=0.2 \mathrm{ml}, S E=0.05)$ and novel $(M=0.2 \mathrm{ml}, S E=$ 0.12 ) control groups was negligible by the end of testing.

A larger than usual number of animals (10 out of 48) failed to drink consistently within the first 4 days of testing and were excluded from the study. Because the animals were distributed equally between the home and novel test environments, the exclusion of these animals did not appear to bias the results. A likely explanation for the lower sampling rate in the present study was that the present subjects were tested with the room lights fully on. Unpublished work in our lab has shown that rats are slower to sample solutions when the solutions are presented in full room light than when they are presented in dim light. In addition, there is evidence to suggest that bright light reduces the rat's tendency to eat in a freefeeding environment (Strubbe, Spiteri, \& Alingh Prins, 1986).

The present results suggest that the rat is also more cautious about food intake in a novel, brightly illuminated environment than when the same food is found in a more familiar environment. Although differences between sucrose intake in the one test environment and sucrose intake in the other did approach significance, the reduction in saccharin intake cannot be explained simply as a general reduction in feeding in the novel test environment, because the $0.15 \%$ saccharin intake for both the novel-tested and home-tested control groups did not differ. Instead, the suppression of saccharin intake in the novel environment depended on the presence of an alternative food. Thus, the results suggest that rats are more selective about intake when alternative food sources are found in an unfamiliar location than when the same foods are found in a familiar location.

\section{EXPERIMENT 3}

Experiment 2 showed that some characteristics of the test situation can contribute to the degree of suppression shown towards new foods. Another factor that may contribute to the amount of suppression found in a test situation is the delay time from the end of testing to the daily postsession feeding. For example, Bacotti (1976) has found that the delay time before daily feeding can affect the amount of operant performance obtained within an experimental session. In both of the preceding studies, we delayed the postsession feeding for $90 \mathrm{~min}$ after testing. However, the delay time before postsession feeding was not reported in the Flaherty and Checke (1982) study.

In Experiment 3, we therefore examined the effects of delay time before daily postsession feeding on saccharin and sucrose intake in the anticipatory contrast paradigm. For one set of animals, the daily feeding was delayed until $90 \mathrm{~min}$ after the end of the experimental session, as in the previous two studies. For the other set, the daily feeding was given immediately upon return to the colony after the session (about a 5-min delay interval). Both experimental groups received a $0.15 \%$ saccharin solution in the first access period, a 32-min delay, and 32\% su- 
crose in the second access period. The control groups were given access to a $0.15 \%$ saccharin solution in the first period and water alone in the second access period.

\section{Method}

Subjects. Forty 120-150-day-old experimentally naive female rats were maintained and treated as in Experiment 1 .

Apparatus. The apparatus and testing conditions were similar to those used in Experiment 1.

Procedure. The animals were divided into four groups-two control groups and two experimental groups-equated for body weight. One subject was subsequently excluded from each group for failure to drink. One set of experimental and control groups was assigned to the immediate feeding condition; the second set was assigned to the delayed feeding condition.

After weighing and a 10-min accommodation period, the subjects in each group were given a 5-min period of access to $10 \mathrm{ml}$ of $0.15 \%$ saccharin solution. A 32 -min delay was timed from the end of the saccharin period to the beginning of the second access period. The subjects in the experimental groups then received $5 \mathrm{~min}$ of access to a $32 \%$ sucrose solution. The subjects in the control groups received water only during the second access period. The subjects in the immediate feeding groups were then returned to the colony and fed their daily ration of lab chow (approximately $5 \mathrm{~min}$ after the end of the second test period). The subjects in the delayed feeding groups were returned to the colony but not fed until $90 \mathrm{~min}$ later. All other procedures were identical to those of the previous experiments.

\section{Results and Discussion}

The amount of $0.15 \%$ saccharin intake for each group is shown in Figure 3 across days of testing. An ANOVA for a $2 \times 2$ factorial design was used to compare mean saccharin intake for the last 2 days of testing across the two feeding times (immediate and delayed) and the two solutions (water and $32 \%$ sucrose) available in the second access period. This analysis indicated a significant difference in saccharin intake for feeding time $[F(1,32)=$ $10.78, p<.01]$, a significant difference for test solution $[F(1,32)=9.58, p<.01]$, and a significant time $X$ solution interaction $[F(1,32)=6.08, p<.05]$. A simple main effects comparison of feeding time with respect to solution indicated no significant difference in saccha-

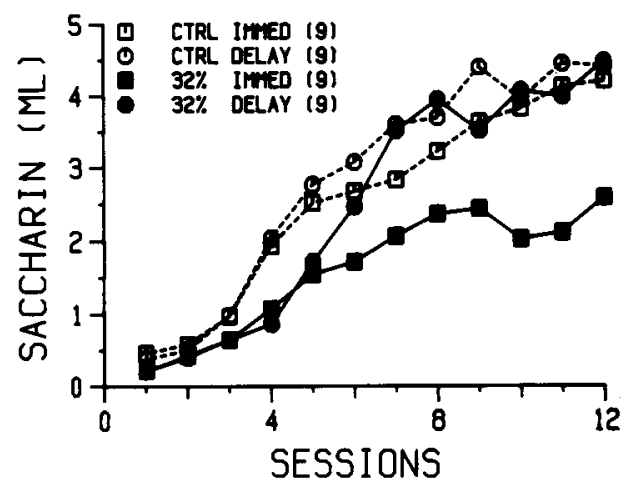

Figure 3. The mean intake of $0.15 \%$ saccharin solution for each group in Experiment 3 across the 12 days of testing. Animals in the immediate feeding condition (squares) were fed 5 min after testing; animals in the delayed feeding condition (circles) were fed $90 \mathrm{~min}$ after testing. rin intake between immediate and delayed feeding for water, but a significant difference between immediate and delayed feeding for $32 \%$ sucrose.

The subjects in the immediate feeding group also consumed less sucrose $(M=9.4 \mathrm{ml}, S E=.48)$ than did those in the delayed feeding group $[M=11.7 \mathrm{ml}, S E=.36$; $F(1,16)=15.17, p<.01]$. Water intake in both the immediate $(M=0.2 \mathrm{ml}, S E=0.07)$ and delayed $(M=$ $0.2 \mathrm{ml}, S E=0.05$ ) feeding control groups was negligible.

One possible interpretation of these results is that the immediate postsession feeding enhanced the value of the $32 \%$ sucrose solution by a classical conditioning process. In this interpretation, pairing the $32 \%$ solution with immediate access to food would serve to increase the "value" of that solution, relative to its value for the group that had delayed feeding. In this manner, the greater suppression found in the immediate feeding condition might be explained by a greater devaluation of the $0.15 \%$ saccharin solution, relative to the greater value of the $32 \%$ sucrose solution in that group.

However, the suppression of $32 \%$ sucrose intake in the immediate feeding group does not appear to be consistent with this hypothesis. If the value of the $32 \%$ sucrose solution was increased by the immediacy of the postsession feeding, then intake of the $32 \%$ solution should also have increased. Instead, intake of both the $32 \%$ sucrose and the $0.15 \%$ saccharin was suppressed when $32 \%$ sucrose was followed by food. This finding would seem to imply that the suppression of intake of both incentives was largely due to anticipation of the postsession feeding. However, no suppression was obtained if the immediate feeding was preceded by water alone. Thus, in the present situation, the presentation of the $32 \%$ sucrose solution in the test situation was necessary, but not sufficient, to produce suppression of intake with a 32-min delay.

\section{GENERAL DISCUSSION}

The present experiments indicate that the time horizon over which an animal appears to evaluate alternative foods is influenced by several variables. In Experiment 1, we found no evidence of suppression of $0.15 \%$ saccharin intake when subsequent access to $32 \%$ sucrose was delayed by $32 \mathrm{~min}$. However, in Experiment 2, saccharin intake in a novel well-lighted test environment was suppressed when subsequent access to $32 \%$ sucrose was delayed by $32 \mathrm{~min}$, but not when the same solutions were presented in the animals' home cages. These findings imply that the amount of suppression of intake of a food is not simply a function of the delay time before an alternative food source is made available, but that the conditions of the testing situation also influence the rat's tendency to suppress intake.

In Experiment 3, we found that the time between testing and the subsequent postsession feeding can also affect experimental estimates of the rat's time horizon. When $32 \%$ sucrose was presented $32 \mathrm{~min}$ after access to $0.15 \%$ saccharin, and postsession feeding was delayed 
another $90 \mathrm{~min}$, saccharin intake did not differ from that of a control group receiving water alone in the second access period. However, when $32 \%$ sucrose was also followed by the animal's primary daily access to food, then saccharin intake was significantly reduced compared to that of the control. These results suggest that the suppression of saccharin intake obtained across longer intervals may depend on both the quality and the amount of food available in the subsequent access period.

From a methodological perspective, the delay time before postsession feeding is a potential confounding variable in many studies of this type. In effect, the postsession feeding period can be considered a third alternative patch. The results of Experiment 3 suggest that a delay of $32 \mathrm{~min}$ between the test situation and the postsession feeding period may not be adequate to isolate fully the effects of the test session feeding periods from the subsequent postsession feeding, under some conditions.

From a functional perspective, the present findings indicate that response competition is not necessary to suppress intake when future food is anticipated. Timberlake et al. (1987) proposed that local competition between times spent investigating alternative food sources could account for most of the suppression of intake obtained when food was presented in two alternative locations. In the present procedure, however, the alternative sources were presented in the same location, yet the animals suppressed intake across the same or longer time frames.

More importantly, the current results indicate that the time horizon over which the rat chooses among alternative resources is not simply based on a fixed time frame of anticipation. Instead, the results suggest that the effective time horizon results from the interaction of anticipatory mechanisms with other factors, such as fear or a combination of hedonic and systemic effects, that may raise or lower the animal's immediate threshold for activity in a particular setting. The present findings extend the estimate of the rat's time horizon to at least $32 \mathrm{~min}$ under some conditions. However, this time frame would still appear to be well below that necessary to account for optimal foraging behavior across a $24-\mathrm{h}$ day.

\section{REFERENCES}

BAcotTI, A. V. (1976). Home cage feeding time controls responding under multiple schedules. Animal Learning \& Behavior, 4, 41-44. Flaherty, C. F., \& CheCKe, S. (1982). Anticipation of incentive gain. Animal Learning \& Behavior, 10, 177-182.

Flaherty, C. F., Rowan, G. A. (1985). Anticipatory contrast: Within-subjects analysis. Animal Learning \& Behavior, 13, 2-5.

FlaherTy, C. F., Rowan, G. A. (1986). Successive, simultaneous, and anticipatory contrast in the consumption of saccharin solutions. Joumal of Experimental Psychology: Animal Behavior Processes, 12, 381-393.

Kamil, A. C., \&oitblat, H. L. (1985). The ecology of foraging behavior: Implications for animal learning and memory. Annual Review of Psychology, 36, 141-169.

LEA, S. E. G. (1981). Correlation and contiguity in foraging behavior. In P. Harzem and M. D. Zeiler (Eds.), Advances in the analysis of behavior: Vol. II. Predictability, correlation, and contiguity (pp. 355406). New York: Wiley.

Pyke, G. H., Pulliam, H. R., \& Charnov, E. L. (1977). Optimal foraging: A selective review of theory and tests. Quarterly Review of Biology, 52, 137-154.

Strubre, J. H., Spiteri, N. J., \& Alingh Prins, AB. J. (1986). Effect of skeleton photoperiod and food availability on the circadian pattern of feeding and drinking in rats. Physiology \& Behavior, 36, 647-651.

Timberlake, W., Gawley, D. J., \& Lucas, G. A. (1987). Time horizons in rats foraging for food in temporally separated patches. Joumal of Experimental Psychology: Animal Behavior Processes, 13, 302-309.

(Manuscript received February 18, 1988; accepted for publication June 27, 1988.) 